\title{
Quantum Theory of Gravitation: General Formulation and Linearized Theory*
}

\author{
R. ARnowitT, $\dagger$ Department of Physics, Syracuse University, Syracuse, New York \\ AND \\ S. Deser, $\ddagger$ Department of Physics, Harvard University, Cambridge, Massachusetts
}

(Received September 11, 1958)

\begin{abstract}
The problem of quantizing general relativity using the Schwinger action principle is considered. The advantages of this technique are discussed and the general formulation of the action principle using the Palatini Lagrangian is given. The difficulty in quantizing general relativity is due to the constraint equations. Two types of constraints are distinguished: algebraic constraint equations and differential constraint equations. The former may be dealt with trivially in this formalism. The latter arise due to the presence of function-type ("gauge") group invariances. In order to eliminate these variables one must make use of the group transformations themselves. Thus in general relativity the transformation from the full set of variables to the independent canonical ones is a coordinate transformation. The linearized theory is treated in detail from this viewpoint and the full theory is briefly discussed.
\end{abstract}

\section{INTRODUCTION}

$\mathrm{A}^{\mathrm{H}}$ LTHOUGH the general theory of relativity has been the subject of considerable investigation and application since its conception, it has been treated to a large extent in a manner apart from the other field theories of physics. The reasons for this lie in two fundamental characteristics of the Einstein theory, namely, the identification of the field variables of the theory with the metric structure of physical space-time on the one hand, and the thoroughly nonlinear nature of the field equations on the other. Further, the historical applications of the theory have tended to stress the static solutions rather than the field as a dynamical entity.

Modern techniques for the treatment of classical or quantum fields have dealt with them as systems of infinitely many degrees of freedom to be investigated by Lagrangian or Hamiltonian formalisms. For the simpler Lorentz-covariant field theories where either no constraint variables exist or alternatively the constraint variables may be eliminated by algebraic manipulation, the canonical treatment follows simply that of ordinary particle mechanics. In the case of fields such as the electromagnetic one where the existence of a gauge group implies that constraints exist, which cannot be so eliminated, the analysis becomes more complicated. For these cases, the problem resides in obtaining an explicit determination of the true dynamical field variables from among the total number required for relativistic invariance. The identification of these canonical variables is most immediate when the field equations are cast in first-order Hamiltonian form. It is well known that any Lorentz-covariant field of integral as well as half-

\footnotetext{
* The writing of this paper was done while both authors enjoyed the hospitality of the working conference on general relativity and elementary particles in Switzerland, summer of 1958, sponsored by Wright Air Development Command, United States Air Force.

$\dagger$ Supported in part by a National Science Foundation research grant.

$\ddagger$ Now at Physics Department, Brandeis University, Waltham, Massachusetts.
}

integral spin can be put in such a Kemmer form. ${ }^{1}$ While it may seem purely a matter of preference as to whether one formulates a set of field equations in first-order or second-order form, it is essential, as already mentioned, even in the classical theory, to utilize first-order equations in order to determine what variables are truly canonical. Further, in the quantum theory, it becomes even more necessary to make this choice. This fact has been stressed by Schwinger ${ }^{2}$ in his formulation of quantization which we shall employ here. The advantage of doing so resides in the rigid connection this procedure establishes between field equations and commutation relations among the canonical variables.

The usual Lagrangian formulation of general relativity views the metric tensor, $g_{\mu \nu}$, as the only field variable. The conventional Lagrangian is then quadratic in the first derivatives of $g_{\mu \nu}$, yielding, upon variation, the second-order Einstein field equations. Such a Lagrangian leads to difficulties in the determination of the canonical variables for the reasons mentioned above. The large amount of nonlinearity of the theory leads to a further difficulty at the quantum level in that there exists many admissible Hermitian quantum Lagrangians which may be formed from the classical one by rearranging the order of the operators. Both these problems can be circumvented by making use of the Palatini ${ }^{3}$ formulation of the Einstein theory. This technique, by making the metric tensor and the affinity, $\Gamma^{\alpha}{ }_{\mu \nu}$, independent field variables reduces the Lagrangian to a structure linear in their derivatives. The relation between the metric and the affinity now results as an extra set of field equations. Thus the Palatini method plays the role of the Kemmer formalism for general

1 O. Klein, Arkiv Mat. Astron. Fysik 25A, No. 15 (1937); N Kemmer, Proc. Roy. Soc. (London) A173, 91 (1939).

${ }^{2}$ J. Schwinger, Phys. Rev. 82, 914 (1951); Phys. Rev. 91, 713 (1953); and lecture notes, Stanford University, 1957 (unpublished).

${ }^{3}$ See, for example, E. Schrödinger, Space-Time Structure (Cambridge University Press, Cambridge, 1950). 
relativity and hence is admirably suited for use in the Schwinger action principle.

Returning to the question of finding the correct canonical variables, the difficulty of reduction from the full number of field quantities (now fifty in number) resides in the invariance of the theory under a function group. In electrodynamics, this group is the gauge group while in general relativity it is, of course, the group of general coordinate transformations involving four independent functions. The decrease in the number of independent variables is implicitly contained in the differential constraints not involving time derivatives (thus the equation $\boldsymbol{\nabla} \cdot \mathbf{E}=0$ in electrodynamics eliminates the longitudinal photons). The viewpoint to be taken in the present work is that the "gauge" groups themselves may be utilized to extract the true canonical variables from among the original variables. Thus starting from an arbitrary "gauge" frame the procedure involves making a "gauge" transformation to that particular frame in which all variables are canonical. In electrodynamics this corresponds to making a transformation to the radiation gauge, which owes its significance to the fact that the Maxwell equations take on pure Hamiltonian form. While the dynamical variables will have been arrived at by going to a special gauge, it should be emphasized that these variables are gauge scalars. In the gravitational case, the corresponding operation is a coordinate transformation to a particular frame. These points will be elucidated explicitly in the following work.

In Sec. 2 the action principle formulation for general relativity in the Palatini scheme will be discussed. Section 3 will be concerned with the linearized gravitational theory; the analogies to electromagnetism will be examined. Section 4 will give a preliminary discussion of the full gravitational theory as well as of specific differences that may be expected between the classical and quantum theory of this field. The detailed treatment of the Einstein theory will be given in a subsequent paper in collaboration with C. W. Misner.

\section{ACTION PRINCIPLE FOR THE PALATINI FORM OF GENERAL RELATIVITY}

The Schwinger action principle ${ }^{2}$ for relativistic fields may be written in the following form ${ }^{4}$

$$
\delta\left\langle a^{\prime} \sigma_{1} \mid b^{\prime \prime} \sigma_{2}\right\rangle=i\left\langle a^{\prime} \sigma_{1}\left|\delta W_{12}\right| b^{\prime \prime} \sigma_{2}\right\rangle
$$

where the action, $W_{12}$, is a four-dimensional integral between space-like surfaces $\sigma_{1}$ and $\sigma_{2}$,

$$
W_{12}=\int_{\sigma_{2}}^{\sigma_{1}} d^{4} x L(x)
$$

${ }^{4}$ We employ natural units $\hbar=c=1$. Further we set $\kappa=8 \pi \gamma / c^{4}=1$ ( $\gamma$ is the Newtonian gravitational constant). This sets the scale of length. Throughout this paper Greek indices run from 0 to 3 , Latin indices from 1 to 3 , and $x^{0}=t$. A comma in a subscript or the symbol $\partial_{\nu}$ will denote an ordinary partial derivative. $a^{\prime}$ and $b^{\prime}$ represent eigenvalues of two complete sets of operators on the respective surfaces $\sigma_{1}$ and $\sigma_{2}$. The Lagrange density, $L(x)$, is to be written in a form linear in the derivatives of the field variables. The general form assumed is

$L(x)=\frac{1}{2}\left[\chi(x) B^{\mu} \partial_{\mu} \chi(x)-\partial_{\mu} \chi(x) B^{\mu} \chi(x)\right]-H\left(\chi_{a}\right)$.

In Eq. (2.3), $\chi$ is a column symbol whose components, $\chi_{a}$, are the field variables (in the first-order representation), $B^{\mu}$ are a set of four numerical matrices (unique for every field) and $H\left(\chi_{a}\right)$ is a scalar density function of the field variables $\chi_{a}$ (but not of their derivatives). Variation of the transformation function on the left hand side of Eq. (2.1) consistent with the dynamics gives rise in general to two types of terms: first a change of the complete set $\{a\}$ on the surface and second variations of the surface corresponding to the space-time displacement of the system. Thus

$$
\delta\left\langle a^{\prime} \sigma_{1} \mid b^{\prime \prime} \sigma_{2}\right\rangle=i\left\langle a^{\prime} \sigma_{1}\left|G\left(\sigma_{1}\right)-G\left(\sigma_{2}\right)\right| b^{\prime \prime} \sigma_{2}\right\rangle,
$$

where $G(\sigma)$ is the generator of the two types of variation. These are represented in the Hilbert space by infinitesimal unitary transformations. In general, then,

$$
G(\sigma)=G_{\chi}(\sigma)+G_{x}(\sigma),
$$

where $G_{\chi}(\sigma)$ generates the changes in the complete set on the surface $\sigma$ and $G_{x}(\sigma)$ generates the space-time displacements. From Eqs. (2.1) and (2.4) one sees that the dynamics of the system is specified by

$$
\delta \int_{\sigma_{2}}^{\sigma_{1}} L(x) d^{4} x=G\left(\sigma_{1}\right)-G\left(\sigma_{2}\right) .
$$

The vanishing of the variation of the action in the interior region between $\sigma_{2}$ and $\sigma_{1}$ [which is contained in Eq. (2.6)] gives rise to the Lagrange equations of motion. As may be seen from the structure of the Lagrange density in Eq. (2.3), these equations are of the Kemmer form. Thus the action principle yields three results: the Lagrange equations of motion and the two generators; these must be internally consistent with one another. From the generator, $G_{x}(\sigma)$, one may define the energy-momentum vector of the system, $P^{\mu}$, which allows one to derive the Heisenberg (commutator) equations of motion. One employs the generator $G_{\chi}(\sigma)$ to obtain the canonical commutation relations in such a fashion that the Heisenberg and Lagrange equations are identical. Thus for any Lagrangian, the Schwinger action principle yields a unique set of commutation relations whose consistency with the equations of motion is a test of the quantizability of the theory. Further, it should be stressed that the principle involves only quantum operators and does not make use, therefore, of the correspondence principle.

The Palatini Lagrange density in general relativity is given by

$$
\begin{array}{r}
L(x)=-\mathfrak{g}^{\mu \nu} \cdot R_{\mu \nu}=\mathfrak{g}^{\mu \nu} \cdot\left[\Gamma^{\alpha}{ }_{\mu \nu, \alpha}-\frac{1}{2}\left(\Gamma_{\mu \alpha, \nu}^{\alpha}+\Gamma_{\nu \alpha, \mu}^{\alpha}\right)\right. \\
-\mathfrak{g}^{\mu \nu} \cdot\left[\left(\Gamma_{\alpha \mu}^{\beta} \cdot \Gamma_{\nu \beta}^{\alpha}\right)-\left(\Gamma_{\alpha \beta}^{\beta} \cdot \Gamma^{\alpha}{ }_{\mu \nu}\right)\right]
\end{array}
$$


where $\mathfrak{g}^{\mu \nu}$ is the metric tensor density, $\Gamma^{\alpha}{ }_{\mu \nu}$ is the affinity, and

$$
A \cdot B \equiv \frac{1}{2}(A B+B A) .
$$

In Eq. (2.7) the fundamental field quantities are $g^{\mu \nu}$ and $\Gamma^{\alpha}{ }_{\mu \nu}$. These quantities are to be varied independently. Note that it is the metric tensor density that is the basic field variable in contradistinction to the usual treatment in which the metric tensor, $g_{\mu \nu}$, is taken as primary. The symmetrization introduced into $L$ insures its Hermiticity. Further,

$$
\begin{aligned}
R_{\mu \nu}=-\Gamma_{\mu \nu, \alpha}^{\alpha}+\frac{1}{2}\left(\Gamma_{\mu \alpha, \nu}^{\alpha}\right. & \left.+\Gamma^{\alpha}{ }_{\nu \alpha, \mu}\right) \\
& +\Gamma^{\beta}{ }_{\alpha \mu} \cdot \Gamma_{\nu \beta}^{\alpha}-\Gamma_{\alpha \beta}^{\beta} \cdot \Gamma_{\mu \nu}^{\alpha},
\end{aligned}
$$

is still a second-order tensor (the symmetrization does not disturb the transformation properties but merely guarantees Hermiticity). There exist other possible Hermitian quantum generalizations of the classical Lagrangian. These, however, cannot be written in the form $\mathrm{g}^{\mu \nu} \cdot R_{\mu \nu}$. In any event, as will be indicated later, all these Lagrangians yield the same Lagrange equations of motion and the same $G_{\chi}(\sigma)$ but perhaps different $G_{x}(\sigma)$. Since the Heisenberg equations of motion are obtained from $G_{x}(\sigma)$, the question of order of factors is to be settled by invoking the consistency between the Heisenberg and the Lagrange equations.

Addition of a divergence to $L(x)$ allows it to be put into the standard form (2.7) where $\chi$ is a fifty-component column symbol, $\chi=\left(\mathfrak{g}^{\mu \nu}, \Gamma_{\rho \sigma}^{\alpha}\right)$, and $H\left(\chi_{a}\right)$ is the cubic structure in Eq. (2.7) (with positive sign). Thus the Lagrangian has the form of a usual Lorentz-covariant theory with cubic interaction. The numerical matrices $B^{\mu}$ are clearly real and antisymmetric. As has been shown by Schwinger, ${ }^{5}$ fields whose $B^{\mu}$ have the above properties, which are Lorentz-covariant, and which possess a lowest energy state, obey Bose-Einstein statistics. Thus in the present case, the intrinsic field variations $\delta \mathfrak{g}^{\mu \nu}$ and $\delta \Gamma^{\alpha}{ }_{\mu \nu}$ commute with all field quantities. It is for this reason that the various possible symmetrizations of the Lagrangian yield identical Lagrange equations and $G_{\chi}(\sigma)$ since variations of the cubic term can produce but one Hermitian quadratic structure.

The equations of motion resulting from $L(x)$ are

$$
\begin{gathered}
R_{\mu \nu}\left(\Gamma^{\alpha}{ }_{\rho \sigma}\right)=0 \\
\mathfrak{g}_{; \alpha}^{\mu \nu} \equiv \mathfrak{g}^{\mu \nu}{ }_{, \alpha}+\mathfrak{g}^{\mu \rho} \cdot \Gamma_{\rho \alpha}^{\nu}+\mathfrak{g}^{\rho \nu} \cdot \Gamma_{\rho \alpha}^{\mu}-\mathfrak{g}^{\mu \nu} \cdot \Gamma_{\alpha \beta}^{\beta}=0 .
\end{gathered}
$$

The second of these equations yields the usual relation between affinity and metric in classical theory. Upon substitution of the Christoffel symbol for $\Gamma^{\alpha}{ }_{\rho \sigma}$ into the first equation one then obtains the usual classical Einstein field equations. The two sets of equations actually obtained correspond to the two sets of Hamilton equations in ordinary mechanics and are likewise of first order in the time derivatives.

${ }^{5}$ J. Schwinger, Proc. Natl. Acad. Sci. U. S. 44, 223, 617 (1958).

\section{LINEARIZED GRAVITATIONAL THEORY ${ }^{6}$}

As a preliminary to treating the full gravitational theory, we consider in this paper the simpler example of the linearized theory in which arise many of the concepts there required. In this section we shall often stress the analogy between gravitation theory and the known electromagnetic results. The Lagrangian for the linearized theory reduces to

$$
\begin{aligned}
L(x)=\gamma^{\mu \nu}\left[\Gamma_{\mu \nu, \alpha}^{\alpha}-\right. & \left.\frac{1}{2}\left(\Gamma_{\mu \alpha, \nu}^{\alpha}+\Gamma_{\nu \alpha, \mu}^{\alpha}\right)\right] \\
& -\eta^{\mu \nu}\left[\Gamma^{\beta}{ }_{\alpha \mu} \cdot \Gamma^{\alpha}{ }_{\nu \beta}-\Gamma_{\alpha \beta}^{\beta} \cdot \Gamma_{\mu \nu}^{\alpha}\right],
\end{aligned}
$$

where $\eta^{\mu \nu}$ is the Lorentz metric (and is thus not a variable) and $\gamma^{\mu \nu}$ is the first-order deviation of $\mathrm{g}^{\mu \nu}$ from $\eta^{\mu \nu}$. Equation (3.1) follows directly from Eq. (2.7) by expanding $\mathfrak{g}^{\mu \nu}$ to first order and noting that $\Gamma^{\alpha}{ }_{\mu \nu}$ has no zeroth-order terms. As may be seen, the linearized theory differs from the full theory only in the reduction of the cubic $H(\chi)$ term to a quadratic form corresponding to the lack of self-interaction of the linearized field. In this section raising and lowering of indices will be done with the Lorentz metric since this is clearly sufficient. The theory admits a group of infinitesimal coordinate transformations which we also shall call gauge transformations,

$$
\begin{aligned}
\gamma^{\mu \nu} & \rightarrow \gamma^{\mu \nu}+\partial^{\mu} \xi^{\nu}+\partial^{\nu} \xi^{\mu}-\eta^{\mu \nu} \partial_{\alpha} \xi^{\alpha} \\
\Gamma_{\mu \nu}^{\alpha} & \rightarrow \Gamma_{\mu \nu}^{\alpha}-\partial_{\mu \nu} \xi^{\alpha}
\end{aligned}
$$

where $\partial_{\mu} \equiv \partial / \partial x^{\mu}, \partial^{\mu} \equiv \eta^{\mu \nu} \partial_{\nu}$, and $\partial_{\mu \nu} \equiv \partial^{2} / \partial x^{\mu} \partial x^{\nu}$. Equations (3.2a) and (3.2b) represent the first-order part of the general coordinate transformations when the gauge functions, $\xi^{\mu}$, are viewed as first-order quantities. Thus if $\bar{x}^{\mu}=x^{\mu}+\xi^{\mu}$ is an infinitesimal coordinate transformation, then

$$
\partial \bar{x}^{\mu} / \partial x^{\nu}=\delta^{\mu}{ }_{\nu}+\partial_{\nu} \xi^{\mu} .
$$

Finally, $\gamma^{\mu \nu}$ and $\Gamma^{\alpha}{ }_{\mu \nu}$ are second- and third-order tensors, respectively, under Lorentz transformations.

In line with the general theory, the action principle yields the equations of motion and the two generating functions. These are, respectively,

$$
\begin{gathered}
-\Gamma_{\mu \nu, \alpha}^{\alpha}+\frac{1}{2}\left(\Gamma_{\mu \alpha, \nu}^{\alpha}+\Gamma_{\nu \alpha, \mu}^{\alpha}\right)=0, \\
-\eta^{\beta \nu} \Gamma_{\beta \alpha}^{\mu}-\eta^{\mu \beta} \Gamma_{\beta \alpha}^{\nu}+\eta^{\mu \nu} \Gamma_{\alpha \beta}^{\beta}=\gamma_{, \alpha}^{\mu \nu}, \\
G_{\chi}=\int d^{3} r\left[\gamma^{\mu \nu} \delta \Gamma_{\mu \nu}^{0}-\gamma^{0 \nu} \delta \Gamma_{\nu \lambda}^{\lambda}\right], \\
G_{x}=\int d^{3} r T^{0 \nu} \delta x_{\nu},
\end{gathered}
$$

${ }^{6}$ The linearized theory has been treated independently along similar lines by J. Schwinger (private communication to S. D.). We should like to thank Professor Schwinger for showing us the results of his work. 
where $^{7}$

$$
\begin{aligned}
& T^{\mu \nu}=\eta^{\mu \nu} L-\frac{1}{2}\left[\gamma^{\alpha \lambda} \Gamma_{\alpha \lambda, \sigma}^{(\mu} \eta^{\nu) \sigma}-\gamma^{(\mu \lambda} \eta^{\nu) \sigma} \Gamma_{\lambda \alpha, \sigma}^{\alpha}\right. \\
& \left.-\gamma^{\alpha \lambda}{ }_{\sigma} \Gamma^{(\mu}{ }_{\alpha \lambda} \eta^{\nu) \sigma}+\gamma^{\lambda(\mu}{ }_{, \sigma} \eta^{\nu) \sigma} \Gamma^{\alpha} \lambda \alpha\right] \\
& -\left(E^{[\sigma \nu] \mu}+E^{[\sigma \mu] \nu}\right), \sigma
\end{aligned}
$$

$$
\begin{aligned}
E^{[\sigma \mu] \nu}=2 \eta^{\tau[\sigma} \gamma^{\mu] \rho} \Gamma^{\nu}{ }_{\tau \rho}+\gamma^{\nu[\sigma} \eta^{\mu] \tau} \Gamma^{\beta}{ }_{\tau \beta} \\
\\
-\frac{1}{2}\left(\gamma^{\tau \rho} \eta^{\nu[\sigma} \Gamma^{\mu]}{ }_{\tau \rho}+\eta^{\nu[\sigma} \gamma^{\mu] \tau} \Gamma^{\beta}{ }_{\tau \beta}\right) .
\end{aligned}
$$

In Eq. (3.6b), the notation "[ [ ]" in the superscript means the antisymmetric part of the tensor and "( )" implies the symmetric part. The integrals in Eqs. (3.5) and (3.6) on the right-hand side are over space-like surfaces which have been chosen to be $t=$ constant for convenience. $\delta x_{\nu}$ is an arbitrary constant space-time translation and rotation.

These results are analogous to the known ones obtained in electrodynamics ${ }^{2}$ when viewed as a first-order theory. Here the Lagrangian is given by

$$
L(x)=\frac{1}{2}\left[A_{\nu} \cdot \partial_{\mu} F^{\mu \nu}-\partial_{\mu} A_{\nu} \cdot F^{\mu \nu}\right]+\frac{1}{4} F^{\mu \nu} F_{\mu \nu}
$$

where $F^{\mu \nu}=-F^{\nu \mu}$ and $A_{\mu}$ are to be varied independently. One obtains from Eq. (3.7) the following results:

$$
\begin{gathered}
\partial^{\mu} F_{\mu \nu}=0, \\
\partial_{\mu} A_{\nu}-\partial_{\nu} A_{\mu}=F_{\mu \nu}, \\
G_{\chi}=\int d^{3} r \frac{1}{2}\left[F_{0 k} \delta A_{k}-A_{k} \delta F_{0 k}\right], \\
G_{x}=\int d^{3} r\left(T^{0 \nu}\right)_{\mathrm{em}} \delta x_{\nu},
\end{gathered}
$$

where $\left(T^{\mu \nu}\right)_{\mathrm{em}}$ is the electromagnetic stress-energy tensor. The equations of motion can be split into two parts, i.e., those involving time derivatives and the constraint equations:

$$
\begin{aligned}
\partial_{0} A_{k} & =F_{0 k}+\partial_{k} A_{0}, \\
\partial_{0} F_{0 k} & =\partial_{j} F_{j k}, \\
F_{j k} & =\partial_{j} A_{k}-\partial_{k} A_{j}, \\
\partial_{j} F_{j 0} & =0 .
\end{aligned}
$$

Equation (3.12a) represents the simple, algebraic type of constraint which allows one to eliminate the magnetic field in terms of the vector potential. Equation (3.12b) is the differential constraint that arises because of gauge invariance. For this case the full content of this differential equation is, of course, that the electric field is transverse. Thus, writing

$$
A_{k}=A_{k}{ }^{T}+\partial_{k} \Lambda(x), \quad \partial_{k} A_{k}{ }^{T}=0,
$$

where $\Lambda(x)$ is arbitrary, one sees that Eqs. (3.11)

\footnotetext{
${ }^{7}$ In deriving the stress-energy tensor of Eqs. (3.6), the symmetrical form of the Lagrangian, Eq. (2.3), was used.
}

decompose into purely Hamiltonian form

$$
\begin{aligned}
\partial_{0} \mathbf{A}^{T} & =-\mathbf{E}, \\
\partial_{0} \mathbf{E} & =-\boldsymbol{\nabla} \times\left(\boldsymbol{\nabla} \times \mathbf{A}^{T}\right) .
\end{aligned}
$$

Similarly, it is easy to see that the generating functions depend only on $\mathbf{A}^{T}$ and $\mathbf{E}$. Thus all physical quantities of the theory depend on the two pairs of canonical variables $\mathbf{A}^{T}$ and $\mathbf{E}$. The commutation relations between the canonical variables now can be obtained easily and shown to be consistent with the generators, $P_{\mu}$, of spacetime translations.

In general the vector potential may be written as

$$
A_{\mu}=A_{\mu}{ }^{D}+\partial_{\mu} \Lambda(x),
$$

where $A_{\mu}{ }^{D}=\left(0, \mathbf{A}^{T}\right)$. Equation (3.15) represents the gauge transformation from an arbitrary gauge to the radiation gauge. Thus the radiation gauge plays a central role in defining the dynamical coordinates (for example, the $A_{\mu}$ in the Lorentz gauge would not be canonical variables). On the other hand, it should be stressed that $\mathbf{A}^{T}$ is a gauge scalar ${ }^{8}$ and thus the dynamical Eqs. (3.14) are gauge invariant.

In the linearized gravitational theory, we again decompose the Lagrange Eqs. (3.4) into constraint equations and those involving time derivatives. The equations relating the affinity and the metric may be recast as follows:

$$
\begin{aligned}
& \partial_{0} h_{i j}=-2 \Gamma_{i j}^{0}+\left(h_{0 i, j}+h_{0 j, i}\right), \\
& \partial_{0} h^{0 \mu}=-\delta^{\mu i}\left(h_{00, i}-\Gamma^{i}{ }_{00}\right)+2 \delta^{\mu 0} \Gamma^{0}{ }_{00}, \\
& \Gamma^{i}{ }_{j k}=-\frac{1}{2}\left(h_{i j, k}+h_{i k, j}-h_{j k, i}\right), \\
& \Gamma^{\mu}{ }_{i 0}=\delta^{\mu k}\left(-h_{0 k, i}+\Gamma^{0}{ }_{i k}\right)+\delta^{\mu 0} \frac{1}{2} h_{00, i},
\end{aligned}
$$

where $h^{\mu \nu}=g^{\mu \nu}-\eta^{\mu \nu}$ is the first-order deviation of the contravariant metric tensor from its flat-space value. Similarly, the field Eqs. (3.4a) become

$$
\begin{gathered}
\partial_{0} \Gamma_{i j}^{0}=-\frac{1}{2}\left[h_{i j, k k}+h_{k k, i j}\right. \\
\left.-h_{k i, j k}-h_{k j, i k}-h_{00, i j}\right], \\
\Gamma_{i k, k}^{0}-\Gamma_{k k, i}^{0}=0 \\
h_{i j, i j}-h_{i i, j j}=0 .
\end{gathered}
$$

Equations (3.6c, d) represent the algebraic constraints analogous to Eq. (3.12a). They have, in fact, been used in the foregoing to eliminate $\Gamma^{i}{ }_{j k}$ and $\Gamma^{\mu}{ }_{i 0}$ from the other equations. Similarly, Eqs. (3.17b, c) are the differential constraint equations arising from the gauge invariance of the theory. Equations (3.16a) and (3.17a) correspond to Eqs. (3.11a, b) and will yield the canonical equations of motion when the "gauge variables" have been separated away. That Eq. (3.16b) is not a true equation of motion is already to be expected from the

\footnotetext{
${ }^{8} \mathrm{It}$ is clear that the radiation gauge is not a Lorentz-covariant concept and consequently $\mathbf{A}^{T}$ is different in different Lorentz frames. The $\mathbf{A}^{T}$ of two Lorentz frames, however, differ by a canonical transformation which is all that is required.
} 
fact that no equation canonically conjugate to it is to be found in the set (3.17); it will be seen below that this equation is merely the defining equation for $\Gamma^{\mu}{ }_{00}$.

The full content of the constraint equation $(3.17 \mathrm{~b})$ is to restrict $\mathrm{I}^{0}{ }_{i j}$ to the form

$$
\begin{gathered}
\Gamma_{i j}^{0}=\left(\Gamma_{i j}^{0}\right)^{T T}-\partial_{i j} \xi^{0}(x), \\
\partial_{i}\left(\Gamma_{i j}^{0}\right)^{T T}=0, \quad\left(\Gamma_{i i}^{0}\right)^{T T}=0,
\end{gathered}
$$

where $\xi^{0}(x)$ is an arbitrary function. Thus, the three differential constraints eliminate three components of $\Gamma^{0}{ }_{i j}$. Similarly (3.17c) restricts $h_{i j}$ to the form

$$
\begin{gathered}
h_{i j}=\left(h_{i j}\right)^{T T}+\partial_{i} \xi_{j}(x)+\partial_{j} \xi_{i}(x), \\
\left(h_{i i}\right)^{T T}=0, \quad \partial_{i}\left(h_{i j}\right)^{T T} \equiv 0,
\end{gathered}
$$

where $\xi_{i}$ is an arbitrary vector. Here one component of $h_{i j}$ has been eliminated. One may now divide Eqs. (3.16a) and (3.17a) into their transverse and longitudinal parts. The transverse parts are the canonical equations of motion,

$$
\begin{gathered}
\partial_{0}\left(h_{i j}\right)^{T T}=-2\left(\Gamma_{i j}^{0}\right)^{T T}, \\
\partial_{0}\left(\Gamma_{i j}^{0}\right)^{T T}=-\frac{1}{2}\left(h_{i j}\right)^{T T}, k k .
\end{gathered}
$$

The longitudinal parts plus Eq. (3.16b) allow one to write

$$
\begin{aligned}
\Gamma_{\mu \nu}^{\alpha} & =\left(\Gamma_{\mu \nu}^{\alpha}\right)^{D}-\partial_{\mu \nu} \xi^{\alpha}, \\
h_{\mu \nu} & =\left(h_{\mu \nu}\right)^{D}+\partial_{\mu} \xi_{\nu}+\partial_{\nu} \xi_{\mu},
\end{aligned}
$$

where

$$
\begin{aligned}
\left(\Gamma_{\mu \nu}^{\alpha}\right)^{D} & \equiv\left[\left(\Gamma_{i j}^{0}\right)^{T T} ;\left(\Gamma_{j k}^{i}\right)^{T T} \equiv-\frac{1}{2}\left\{\left(h_{i j}\right)^{T T}{ }_{k}+\left(h_{i k}\right)^{T T}{ }_{j}-\left(h_{j k}\right)^{T T}{ }_{i}\right\} ;\left(\Gamma^{k}{ }_{i 0}\right)^{T T} \equiv\left(\Gamma_{i k}^{0}\right)^{T T}\right] \\
\left(h_{\mu \nu}\right)^{D} & \equiv\left(h_{i j}\right)^{T T} .
\end{aligned}
$$

Substituting Eqs. (3.21) into the generator $G_{\chi}$ yields (upon appropriate integration by parts and neglect of a total variation)

$$
G_{\chi}=\int d^{3} r\left(h_{i j}\right)^{T T} \delta\left(\Gamma_{i j}^{0}\right)^{T T}
$$

A lengthier calculation shows that $G_{x}$ also depends only on the dynamical variables. The energy-momentum vector $P^{\mu}$ can be formed from Eq. (3.6) by restricting $\delta x_{\mu}$ to be a translation $\epsilon_{\mu}$,

$$
G_{x}=\epsilon_{\mu} P^{\mu} .
$$

From the fact that $\epsilon_{0} P^{0}=-\epsilon^{0} P^{0}$ is the generator of time translations in the direction $\epsilon^{0}$, one can derive the Heisenberg equations of motion

where

$$
\left[\chi^{D}, P^{0}\right]=i \partial_{0} \chi^{D}
$$

$P^{0}=\int d^{3} r\left[\left(\Gamma^{0}{ }_{i j}\right)^{T T}\left(\Gamma^{0}{ }_{i j}\right)^{T T}+\frac{1}{4}\left(h_{i j}\right)^{T T},{ }_{k}\left(h_{i j}\right)^{T T}, k\right]$

and $\chi^{D}$ is any one of the dynamical variables. Making use of the canonical equations of motion, one finds

$$
\begin{aligned}
{\left[G_{\chi}, P^{0}\right] } & =i \int d^{3} r \partial_{0}\left(h_{i j}\right)^{T T} \delta\left(\Gamma^{0}{ }_{i j}\right)^{T T} \\
& =-2 i \int d^{3} r\left(\Gamma^{0}{ }_{i j}\right)^{T T} \delta\left(\Gamma^{0}{ }_{i j}\right)^{T T}
\end{aligned}
$$

On the other hand, the left-hand side may be viewed as generating a change in $P^{0}$ due to $G_{\chi}$,

$$
\left[G_{\chi}, P^{0}\right]=i \delta_{\Gamma} P^{0}=i\left[P^{0}\left(\Gamma^{T T}-\delta \Gamma^{T T}\right)-P^{0}\left(\Gamma^{T T}\right)\right] .
$$

The second equality in Eq. (3.28) follows from compari- son with the right-hand side of Eq. (3.27). From this we see that $G_{\chi}$ is the generator of unitary transformations which send $\left(\Gamma_{i j}^{0}\right)^{T T}$ into $\left(\Gamma_{i j}^{0}\right)^{T T}-\delta\left(\Gamma_{i j}^{0}\right)^{T T}$. This interpretation arises from the requirement of consistency between the Heisenberg (commutator) equations of motion and the Hamiltonian ones. From this interpretation of $G_{\chi}$ follow the commutation relations. Thus

or

$$
\left[\left(\Gamma^{0}{ }_{i j}\right)^{T T}, G_{\chi}\right]=i \delta\left(\Gamma_{i j}^{0}\right)^{T T},
$$

$$
\begin{array}{r}
{\left[\Gamma_{i j}^{0}(\mathbf{r})^{T T}, h_{k l}\left(\mathbf{r}^{\prime}\right)^{T T}\right]=\frac{1}{2} i[} \\
{\left[\left(\delta_{k i} \delta_{l j}+\delta_{l i} \delta_{k j}\right.\right.} \\
\left.\left.\quad-\frac{2}{3} \delta_{k l} \delta_{i j}\right) \delta\left(\mathbf{r}-\mathbf{r}^{\prime}\right)\right]^{T} .
\end{array}
$$

Similarly one may show that all other equal-time commutators between the canonical variables vanish.

Returning to Eq. (3.21), we note that it is just of the form of the coordinate transformations (3.2). Thus, as in electrodynamics, the separation of the dynamical variables from the redundant total number is achieved by making a gauge transformation to the correct "radiation" gauge. Again, the dynamical variables $\left(\Gamma^{0}{ }_{i j}\right)^{T T}$ and $\left(h_{i j}\right)^{T T}$ are gauge invariant, i.e., scalars under infinitesimal coordinate transformations.

\section{GENERAL THEORY}

In this section a preliminary discussion will be given of the problem of finding the canonical variables in the general theory of relativity and of their quantization. As was seen in the previous section, the difficulty involved in quantizing a theory invariant under a gauge type of group stems from the existence of differential constraints. The elimination of the variables associated with these constraints, however, was performed precisely through use of the gauge invariance inherent in the theory. For the linear theory, this procedure was relatively transparent due to the additive nature of the infinitesimal coordinate transformations. For general 
relativity, the gauge group is the group of arbitrary coordinate transformations and is, of course, multiplicative in nature. However, just as in electrodynamics where, in the full vector potential, the transverse part (a gauge scalar) is isolated, there exists a part of the metric tensor which is coordinate invariant. Thus in the tensor transformation formula

$$
\begin{aligned}
g_{\mu \nu}(x) & =\frac{\partial \xi^{\alpha}}{\partial x^{\mu}} \frac{\partial \xi^{\beta}}{\partial x^{\nu}} g^{\prime}{ }_{\alpha \beta}(\xi), \\
\Gamma^{\alpha}{ }_{\mu \nu}(x) & =\frac{\partial \xi^{\rho}}{\partial x^{\mu}} \frac{\partial \xi^{\tau}}{\partial x^{\nu}} \frac{\partial x^{\alpha}}{\partial \xi^{\sigma}} \Gamma_{\rho \tau}^{\prime}{ }_{\rho \tau}(\xi)+\frac{\partial x^{\alpha}}{\partial \xi^{\beta}} \frac{\partial^{2} \xi^{\beta}}{\partial x^{\mu} x^{\nu}},
\end{aligned}
$$

part of the metric tensor changes but certain combinations of the components of $g_{\mu \nu}$ are scalars. That this is possible can be seen naively from the fact that the four gauge functions, $\xi^{\alpha}$, cannot really affect all ten components of the metric tensor when the proper combinations are chosen. Hence, the problem of finding the canonical variables in general relativity resides in finding the coordinate transformation which leads one to the "radiation" gauge where all the variables are dynamical. The actual treatment of this program will be given in a subsequent paper in collaboration with C. W. Misner.

The preceding approach tends to treat general relativity as a Lorentz-covariant field theory with a gauge invariance. The gauge invariance then summarizes the geometrical interpretation of relativity. However, since this method concentrates on the gauge-independent aspects of the theory, the geometry is kept in the background in comparison with the Lorentz-covariant features. In a certain respect the Lorentz covariance is not merely a formal property but is intrinsically tied to the physical interpretation of the theory. For any field, in general, an essential requirement for the carrying out of quantum measurements is that there exist an apparatus that is not unknowably distorted by the field to be measured. In the gravitational case, because of the universal coupling of the gravitational field to all matter and because of the fact that coordinate frames, being mathematical constructs, cannot be distinguished physically from each other, one must assume that the measuring devices are anchored in a known metric (the simplest example being a Lorentz metric). Thus we assume that at spatial infinity, where the measuring devices are fixed, space is flat. As a further point such physically important quantities as the action integral and the energy-momentum vector, $P^{\mu}$, would not exist unless the space became asymptotically flat.

The action principle furnishes us with a stress-energy tensor and therefore with an energy-momentum vector. These will depend only upon the dynamical variables. In this sense the classical radiation problem becomes well-defined as in electromagnetic theory.

The nonlinear nature of general relativity produces effects in the quantum theory that are different from those found in other fields. Equation (2.11) gives the quantum relation between the affinity and the metric. The anticommutator appearing in this equation will change the usual relation between affinity and Christoffel symbol due to quantum effects. Thus the quantum theory will produce fluctuations outside of the classical Riemannian space. Similar ordering questions will arise in defining the canonical commutation relations. Thus one does not expect that correspondence methods of quantization would in general produce valid results.

The applications of the quantum relativity theory would be expected to lie in the domain of elementary particle theory. In particular, it is to be expected from previous considerations ${ }^{9}$ that the divergence difficulty of Lorentz-covariant theories will be ameliorated. Once the dynamical variables have been isolated, it should be possible to set up a functional integral expression ${ }^{10}$ of the theory involving only these variables to investigate such questions further.

\section{ACKNOWLEDGMENT}

We should like to thank Professor O. Klein for stimulating conversations.

${ }^{9}$ S. Deser, Revs. Modern Phys. 29, 417 (1957).

${ }^{10} \mathrm{~S}$. F. Edwards and R. E. Peierls, Proc. Roy. Soc. (London) A224, 24 (1954); P. T. Matthews and A. Salam, Nuovo cimento 12,563 (1954). 